\title{
À propos de Quelques arpents d'Amérique de Gérard Bouchard
}

\section{Christian Dessureault, Thomas Wien et Gérard Bouchard}

Volume 50, numéro 3, hiver 1997

URI : https://id.erudit.org/iderudit/305572ar

DOI : https://doi.org/10.7202/305572ar

Aller au sommaire du numéro

Éditeur(s)

Institut d'histoire de l'Amérique française

ISSN

0035-2357 (imprimé)

1492-1383 (numérique)

Découvrir la revue

Citer ce document

Dessureault, C., Wien, T. \& Bouchard, G. (1997). À propos de Quelques arpents

d'Amérique de Gérard Bouchard. Revue d'histoire de l'Amérique française, 50(3),

401-435. https://doi.org/10.7202/305572ar d'utilisation que vous pouvez consulter en ligne. 


\section{DÉBAT À PROPOS DE QUELQUES ARPENTS D'AMÉRIQUE DE GÉRARD BOUCHARD}

CHRISTIAN DESSUREAULT THOMAS WIEN Département d'histoire Université de Montréal

GÉRARD BOUCHARD IREP

Université du Québec à Chicoutimi

Le Comité de rédaction a souhaité ce débat et il en a fixé les règles. Christian Dessureault et Thomas Wien ont été invités à préparer une note critique d'environ une douzaine de pages et Gérard Bouchard a accepté de répondre à cette critique dans un texte de même longueur. Il était aussi convenu que les premiers avaient ensuite un droit de réplique de une ou deux pages, tandis que le dernier mot était laissé à l'auteur. Nous remercions sincèrement les trois historiens pour cette stimulante contribution.

Le Comité de rédaction

\section{NOTE CRITIQUE}

Gérard Bouchard, Quelques arpents d'Amérique

Population, économie, famille au Saguenay 1838-1971

Montréal, Boréal, 1996, 635 p.

\section{CHRISTIAN DESSUREAULT THOMAS WIEN}

Parmi les historiens québécois, Gérard Bouchard occupe une place à part. C'est celui qui s'adonne le plus aux plaisirs de la modélisation, celui qui affiche le goût le plus marqué pour l'hypothèse explicite, celui enfin qui fait voyager le plus loin son regard comparatif. Se distinguant aussi au chapitre de l'organisation du travail, il s'est lancé presque à corps perdu dans la recherche «industrielle», enchaînant les 
projets et multipliant équipes et banques de données. Bref, G. Bouchard est l'historien du Québec qui, dans sa façon de penser comme dans celle de travailler, a intégré le plus résolument l'approche des sciences sociales. Et parmi les disciplines qu'il fréquente, c'est la sociologie qui, pour le meilleur ou pour le pire, joue le rôle de première confidente de l'histoire.

Intéressant à titre d'invitation au voyage aux confins de la discipline, le livre paru en mai dernier ne l'est pas moins en tant que rendez-vous différé. Multipliant les sujets d'enquête tout en répondant, sans doute, aux exigences particulières de la recherche subventionnée, G. Bouchard a émis, seul ou en collaboration, un véritable barrage de communications et d'articles, remettant à plus tard la synthèse. La voici. Même sans l'étude socioculturelle réservée pour un ouvrage ultérieur, Quelques arpents d'Amérique est un livre consistant, comme il s'en écrit - ou du moins, s'en publie - peu de nos jours. Permettant de faire le point sur vingt ans de recherches en dixneuf chapitres, le livre est pourtant plus qu'un recueil de rapports d'étape. S'il reprend à peu près tels quels cinq articles publiés antérieurement, d'autres sont enrichis de données nouvelles et de nuances (c'est le cas, notamment, de ceux portant sur la reproduction familiale). Enfin, plusieurs chapitres sont inédits. C'est donc un ouvrage cohérent, à l'argumentation cumulative et, comme toujours, au style élégant, que présente $\mathrm{G}$. Bouchard. En même temps, malgré les ajustements survenus au fil des ans au gré des découvertes et des commentaires de la critique, l'ouvrage reste fidèle aux postulats initiaux. Aussi cette histoire d'une colonisation fait-elle apparaître en filigrane l'histoire, tout aussi passionnante, d'une réflexion de longue haleine.

L'ouvrage comprend quatre grandes parties. La première passe en revue les étapes du peuplement à partir de 1838 avant de tracer les contours de l'économie saguenayenne et de ses rapports avec l'économie englobante. Suit l'étude de la reproduction sociale dans un contexte de peuplement, au cœur à la fois du livre et du propos de G. Bouchard. Dans la troisième partie, l'auteur prend du recul, cherchant des points de repère d'abord dans le comportement des familles ouvrières et ensuite dans l'historiographie française et nord-américaine. La dernière partie nous ramène au Saguenay pour ausculter les mécanismes, plus complexes que prévu, de la fin du système de reproduction.

Aux visées assez vastes, car elle traite non seulement de la mise en place de la société régionale mais aussi de l'économie, la première partie est néanmoins conçue comme une mise en situation. C'est dire toute l'importance que revêt pour l'auteur l'étude sociale de la partie 
suivante. Les deux premiers chapitres sont consacrés à la croissance de la population saguenayenne de 1842 à 1971, et à la mobilité géographique des colons. L'immigration initiale - si l'on excepte celle des Amérindiens, l'aventure saguenayenne se déroulant après tout en territoire innu! - fut d'abord alimentée par le surpeuplement rural du Charlevoix et, dans une moindre mesure, du Bas-Saint-Laurent. Ce mouvement migratoire peut par ailleurs compter sur le développement parallèle de l'industrie forestière ainsi que sur le soutien des élites traditionnelles. Mais, pour l'auteur, c'est la famille qui prime: ces premières migrations vers le Saguenay sont principalement dictées par les impératifs de la reproduction familiale.

La même dynamique impulse la mobilité spatiale à l'intérieur de la région au cours du siècle suivant. De plusieurs façons, les relations de parenté commandent ces déplacements. Dès la fin du second chapitre, G. Bouchard peut ainsi avancer que, dans ce contexte d'instabilité démographique et géographique, «le groupe familial avec ses prolongements dans la parentèle et dans l'entraide locale s'annonce comme un facteur déterminant» (p. 57) de la vie sociale.

Les quatre autres chapitres de la première partie traitent de l'histoire de l'ancienne économie rurale du Saguenay_Lac-Saint-Jean des origines jusqu'aux grands changements des années 1950. Dans le chapitre III, l'auteur présente l'évolution des méthodes et des techniques agricoles dans la région. Il insiste sur l'aspect très rudimentaire de l'outillage agricole, sur la diffusion lente et inégale des innovations et, surtout, sur le décalage persistant entre les zones plus anciennement peuplées de la région et les nouveaux terroirs. Cette dernière constatation n'est pas fortuite, car, pour l'auteur, elle fait partie d'un mouvement plus vaste. Ce concept de régression - celui d'adaptation nous apparaît à la fois plus neutre et moins univoque - veut que la colonisation suscite un certain nombre de blocages, voire de reculs, dans la vie matérielle, dans l'économie agraire et dans la vie sociale et culturelle.

En abordant l'agriculture sous l'angle de sa spécialisation laitière, le chapitre IV étudie de plus près un de ces blocages. C'est du moins l'avis de l'auteur qui voit dans l'industrie laitière, qui s'implante vers 1880 , une occasion manquée de faire sauter le verrou qui emprisonne l'ancienne économie paysanne dans une rationalité typiquement précapitaliste. Ce démarrage raté de l'agriculture saguenayenne, alors que, d'après l'auteur, les conditions objectives auraient dû favoriser l'essor du capitalisme agraire, reposerait sur la résistance socioculturelle d'une paysannerie dont les objectifs prioritaires de reproduction familiale imposent «un modèle longtemps réfractaire aux valeurs ca- 
pitalistes» (p. 82). Les familles paysannes saguenayennes, soutient l'auteur, étaient prêtes à participer à l'économie de marché afin d'améliorer leurs conditions de vie et faciliter leur reproduction familiale. Mais au-delà d'un certain seuil, elles résistaient aux tendances nouvelles de la spécialisation et de la commercialisation de l'agriculture qui «mettaient en péril des pratiques de travail, des objectifs socio-économiques ainsi qu'un mode de vie réfractaire au système capitaliste et aux formes sociales et culturelles qui lui étaient associées» (p. 98).

Le chapitre $\mathrm{V}$ sur le travail forestier présente une autre forme, tout aussi circonscrite, de rapports entre la paysannerie et le capitalisme. Pour G. Bouchard, les familles puisaient dans le travail forestier, à l'instar de l'industrie laitière, des ressources complémentaires utiles à leur développement et à leur reproduction familiale, à l'intérieur même de la logique de l'économie paysanne. La description de l'univers culturel et matériel des chantiers occupe une place importante dans ce chapitre. C'est néanmoins la complémentarité entre l'agriculture et le travail forestier qui ressort comme thème majeur: les revenus de la forêt sont particulièrement utiles dans le démarrage initial de l'agriculture dans les nouveaux terroirs, mais l'apport de l'exploitation forestière au développement d'une agriculture plus commerciale est minime. Le travail forestier favorise ainsi l'implantation et l'expansion d'une économie paysanne relativement autonome sans altérer ni son mode de fonctionnement interne ni le système de valeurs qui s'y greffe.

Que ce soit par le biais de la commercialisation de surplus agricoles (dont la vente de produits laitiers) ou du travail à l'extérieur de l'exploitation agricole (le travail forestier et d'autres formes de «pluriactivité»), l'économie rurale saguenayenne entretient donc des rapports ambivalents avec le capitalisme. Dans le chapitre VI, l'auteur propose un modèle théorique qui formalise cette ambivalence. Après avoir fait la critique des principaux modèles déjà proposés dans l'historiographie sur cette question, il s'appuie sur le cas saguenayen et sur d'autres exemples historiques de pluriactivité rurale pour présenter un nouveau concept: la co-intégration. L'idée de base, peu surprenante puisque les chapitres précédents l'appliquent déjà, est que la paysannerie peut entretenir des relations constantes et étroites avec le marché et le capitalisme extra-régional sans se convertir formellement à ses règles. La famille paysanne reste fidèle à ses propres objectifs qui sont, pour l'essentiel, d'assurer sa reproduction à l'identique et son extension physique dans l'espace sans sacrifier ses valeurs communautaires. En somme, la co-intégration met l'accent sur la réciprocité 
plutôt que sur l'inégalité des rapports entre les deux parties. Elles retirent chacune des bénéfices de leur relation: l'une peut accumuler les richesses tandis que l'autre peut se reproduire et occuper l'espace. Nous y reviendrons.

Tout dans cette première partie prépare le terrain pour l'analyse de la reproduction sociale qui suit. On dirait même que la main invisible de la reproduction intervient dès le départ pour régler les comportements démographiques et économiques des familles paysannes. Dans la seconde partie, la main devient visible, et comment: l'auteur consacre pas moins de sept chapitres à l'étude du mode de reproduction familiale. Dans le chapitre VII, c'est d'abord la présentation des sources qui attire l'attention, car elle permet au lecteur, spécialiste ou non, de saisir l'ampleur de cette vaste enquête. Aucune autre recherche sur la reproduction sociale ne s'appuie sur un corpus aussi large et aussi riche en données, sociodémographiques surtout. Le fichier de population BALSAC permet de reconstituer des milliers de familles paysannes et de les suivre dans l'ensemble de l'espace saguenayen. Des données tirées de diverses autres sources sont jumelées aux fiches de familles. Elles offrent une prise sur certains mécanismes de la reproduction sociale tel le recours à la donation entre vifs. Mais la plupart de ces autres séries fournissent des «marqueurs» (statut professionnel, propriété foncière) facilitant l'évaluation, pour un très grand nombre de cas, de la réussite ou de l'échec de la reproduction sociale.

Ces notions de réussite et d'échec posent d'ailleurs problème et c'est à ce titre que l'auteur leur consacre une bonne partie du volet théorique de ce chapitre. C'est l'occasion, pour lui comme pour nous, de revenir sur une des principales objections des critiques du «modèle Bouchard». L'auteur a ici le mérite d'être très explicite sur sa conception de ce qui constitue, à ses yeux, une reproduction réussie. Sa notion de réussite réfère à la capacité des familles paysannes d'établir sur des terres, dans la stabilité ou dans la mobilité géographique, la proportion la plus élevée possible d'enfants. Cette notion de réussite évacue, du moins dans un premier temps, l'établissement sans le secours de la famille, ainsi que plusieurs sortes d'inégalités: celles liées à la localisation, à la superficie, à la productivité et la valeur des terres disponibles pour la nouvelle génération, entre les membres d'une même famille et, surtout, entre les différentes familles paysannes. G. Bouchard ne nie pas l'existence des inégalités dans la société paysanne et les répercussions sociales différentielles du mode de transmission des biens. Mais, conclusion surprenante, ces inégalités ne lui semblent pas être l'un des facteurs qui conditionnent et déterminent à la fois les modalités de la transmission des avoirs familiaux. 
Il reste que, dans leur simplicité même, les critères de réussite de G. Bouchard offrent l'avantage inestimable de «s'opérationnaliser» facilement à l'aide d'une banque de données familiales. Par là, elles permettent de suivre sur une grande échelle les processus de reproduction. C'est déjà beaucoup. Pour l'essentiel, il s'agit, à partir des dossiers de familles reconstituées, de déterminer la persistance du statut de cultivateur d'une génération à l'autre et de vérifier la possession éventuelle d'au moins une terre. La notion d'échec (= pas de terre) est tout aussi simple et, partant, problématique, puisqu'elle recouvre l'ensemble des destins sociaux, enviables ou non, supposés contraires à la logique interne du système de «pluriétablissement» agricole.

À sa décharge, l'auteur demeure conscient de la nécessité de mener des analyses plus fines, à partir d'une documentation plus détaillée portant nécessairement sur des corpus plus restreints, afin de mieux saisir certaines modalités de la reproduction. Dans les six chapitres subséquents, des recherches empiriques sur des thèmes apparemment distincts contribuent à éclairer davantage le fonctionnement de ce système de reproduction familiale.

Assez technique, le chapitre VIII traite des paramètres démographiques de la société saguenayenne. L'auteur attribue le nombre élevé - mais pas exceptionnellement élevé — d'enfants par famille à la culture de la fécondité, de la parenté et de la continuité typique des régions de peuplement récent. Les enfants représentent à la fois un apport direct de main-d'œuvre, une source éventuelle de revenus complémentaires et le noyau d'un réseau d'entraide communautaire. En contrepartie, ils commandent des coûts additionnels pour l'ensemble des membres de la famille. À chaque génération, l'établissement de cinq ou six enfants représente, bien sûr, un fardeau économique pour la famille paysanne. Elle peut toutefois l'assumer assez facilement tant que la terre demeure abondante et que tous ses membres acceptent de contribuer, d'une manière ou d'une autre, à l'effort communautaire.

Le chapitre IX présente certaines composantes du modèle saguenayen de reproduction sociale: le système ouvert de reproduction typique des régions de peuplement récent. Le contexte de colonisation permettait aux familles paysannes à la fois d'assurer, par le biais de la transmission des avoirs familiaux, la sécurité des parents et l'établissement de la très grande majorité des enfants. Les stratégies mises en œuvre pour assurer ces deux buts comprenaient parfois la cession de la terre principale à un fils donataire, mais le modèle ne privilégiait pas l'intégrité des patrimoines fonciers. Celle-ci était plutôt subordonnée, sinon souvent sacrifiée, à l'objectif principal du pluriétablissement. 
On sait que la transmission des avoirs familiaux n'est pas circonscrite à un moment spécifique de la vie familiale, au moment de la retraite ou du décès des parents. G. Bouchard a le grand mérite d'être, parmi tous les spécialistes de ce champ de recherche, celui qui a le mieux fait ressortir, du moins sur le plan théorique, la complexité du processus de transmission, tant dans les modalités que dans la nature des biens transmis, et son étalement sur plusieurs décennies correspondant à différentes phases du cycle de la vie familiale. L'auteur constate les tendances égalitaires de ces pratiques de transmission à l'intérieur des familles et la discrimination systémique, typique d'une société paysanne patriarcale, pratiquée en faveur des garçons.

L'étape de la donation est sans contredit la phase du processus de reproduction familiale la plus étudiée dans l'historiographie. Une conception restrictive de la transmission des patrimoines a longtemps amené les chercheurs à considérer la donation comme la forme presque exclusive de transmission. Comme le souligne l'auteur, la donation demeure malgré tout un champ d'étude stratégique; elle représente un mode d'établissement fortement chargé d'enjeux tant pour les parents donateurs et l'enfant donataire que pour les autres membres de la famille. G. Bouchard réserve donc une attention particulière aux modalités spécifiques de la donation au Saguenay, étudiées à partir d'un échantillon de 306 donations se rapportant à quatre terroirs distincts.

L'analyse de ce corpus révèle des conditions spécifiques à la période et à la région. Ici, l'auteur compare les pratiques saguenayennes à celles observées ailleurs par Sylvie Dépatie (la situation confortable des donateurs), Béatrice Craig (les objectifs des donateurs et du donataire) et plusieurs chercheurs européens (les répercussions de la donation sur les comportements démographiques). L'auteur constate que ce mode d'établissement était plus efficace que tout autre pour lier le destin social du bénéficiaire à l'agriculture et au monde rural. En même temps, et voilà l'essentiel, les modalités spécifiques de la donation n'altéraient pas de façon substantielle les tendances égalitaires du système de reproduction saguenayen.

Soulignons que, en dépit des conclusions de l'auteur, son étude des donations ne conforte pas sa problématique générale puisque ici la singularité du cas saguenayen ne repose pas nécessairement sur des conditions typiques à une région de peuplement. En effet, d'autres historiens, qui travaillent aussi sur des terroirs en expansion, mais pour d'autres époques, présentent des pratiques de donation différentes de celles du Saguenay. Ajoutons que la manière de traiter le sujet, en isolant la donation des autres pratiques successorales, est d'une 
certaine manière en contradiction avec la conceptualisation générale de G. Bouchard de la transmission comme processus complexe et dynamique où les différentes phases de ce processus sont liées les unes aux autres. Cette nouvelle conceptualisation ne commande-t-elle pas, à son tour, une nouvelle approche méthodologique? Les différentes pratiques successorales constituant un processus global, la compréhension de l'une de ces phases devient largement tributaire des informations relatives aux autres étapes du processus.

Dans le chapitre X, l'auteur s'attarde d'abord à la répartition et à la valeur des terres. Il rappelle la prédominance dans le Saguenay, comme dans l'ensemble du Québec, de la propriété paysanne du sol et de l'exploitation familiale. Par ailleurs, il constate la valeur relativement faible des terres dans les secteurs de colonisation pour en conclure que «le travail et la main-d'œuvre requis pour le défrichement d'une ferme représentait un obstacle nettement plus important que le coût de la terre elle-même» (p. 227), ce qui, selon lui, montre encore une fois le rôle déterminant de la contribution familiale dans l'établissement des fils tantôt sous forme financière pour acquérir une exploitation déjà développée, tantôt sous forme de prestation de travail dans le cas d'une terre en friche.

Une seconde section du chapitre est consacrée à l'analyse approfondie du cycle d'accumulation et de contraction des avoirs fonciers au cours de la vie d'une famille. La famille paysanne agrandit la taille de son patrimoine foncier durant une première phase correspondant, de manière générale, à son extension démographique. Puis, dans une seconde phase, la taille des avoirs fonciers se contracte au fur et à mesure de l'établissement des enfants et du vieillissement des parents. Fidèle à son leitmotiv, G. Bouchard lie l'existence de ce cycle aux objectifs et aux pratiques de la reproduction familiale. L'acquisition de nouvelles terres ne viserait ni l'accumulation ni la concentration du capital foncier, mais uniquement la constitution d'une réserve de terres afin de pourvoir à l'établissement éventuel des enfants (mais ce cycle est-il propre aux paysans ou même aux sociétés pré-industrielles?). Par ailleurs, la phase de redistribution des avoirs fonciers aurait consolidé la co-intégration en favorisant l'expansion physique de la petite exploitation familiale aux dépens de la formation de grandes propriétés foncières qui seraient mieux adaptées à l'économie de marché et au capitalisme.

Le chapitre XI creuse l'aspect spatial d'une reproduction familiale qui s'effectue tantôt dans la sédentarité, tantôt dans la mobilité. Comme l'auteur l'a déjà souligné, l'établissement éventuel des enfants commandait l'expansion des avoirs fonciers. Certains parents plus 
fortunés pouvaient acquérir dans leur voisinage immédiat des terres déjà défrichées et ainsi réunir leurs enfants autour d'eux sans se départir du «vieux bien». Mais les cas d'expansion et de reproduction moyennant un déplacement vers le front de colonisation étaient beaucoup plus courants. C'est donc à cette dynamique de la mobilité et de la réallocation des ressources que $\mathrm{G}$. Bouchard consacre la majeure partie de ce chapitre.

Ce principe de réallocation des ressources consiste pour les familles à se défaire, dans une perspective de reproduction familiale, d'une exploitation située dans un terroir plus ancien, ce qui représente un capital foncier relativement coûteux, pour acquérir dans un terroir de peuplement, plusieurs terres de moindre valeur. Les parents font ensuite appel à la force de travail abondante et sous-utilisée de la famille pour mettre ces terres en valeur et y établir tour à tour leurs enfants. Plutôt qu'un signe d'échec ou de désintégration sociale, les migrations deviennent ainsi le reflet d'une stratégie de reproduction sociale montrant la volonté de familles paysannes de maintenir la proximité résidentielle parents/enfants. Ces stratégies, ajoute l'auteur, «révèlent une certaine conception de la famille et de la communauté à édifier» (p. 255).

Le chapitre XII, sur la formation et la structure de la famille, semble, de prime abord, un peu à la périphérie du thème général de la seconde partie de l'ouvrage. L'auteur y aborde successivement l'étude de l'alliance, de la structure des ménages et de l'organisation d'un réseau communautaire paysan fondé sur des rapports de parenté, à dominante patrilinéaire. G. Bouchard tire toutefois de ces analyses apparemment discrètes des conclusions interreliées qui tendent à la fois à éclairer davantage et à nuancer le «système ouvert» saguenayen.

Dans ce modèle, l'alliance ne constitue pas un vecteur matériel important de la reproduction sociale. La liberté relative dans le choix des conjoints et surtout l'absence apparente de stratégies matrimoniales à visée économique dans les familles le laissent deviner. La prédominance de la structure conjugale des ménages qui, de manière générale, correspond au même type d'unité résidentielle, reflète une certaine forme d'individualisme et d'autonomie typique de l'univers du petit exploitant-propriétaire. Mais ces traits culturels, qui s'expriment davantage comme un facteur de résistance de la société paysanne face aux pressions extérieures (l'encadrement du clergé et de l'État ou l'intégration au marché), s'insèrent dans un réseau familial plus large, de type communautaire. D'où un individualisme assez particulier: «On serait tenté, écrit G. Bouchard, de parler d'une sorte d' 'individualisme' de la famille, dans la mesure où cette instance avait 
priorité, d'un côté, sur les individualités elles-mêmes et, de l'autre, sur la communauté élargie à l'échelle du rang et de la paroisse» (p. 276).

Le chapitre XIII traite «des inégalités, des ruptures, des échecs individuels et familiaux» (p. 277), c'est-à-dire de l'objet controversé des revers dans le système de reproduction saguenayen. Mais l'auteur prend soin de bien circonscrire la nature des revers appréhendés: encore une fois, l'étude ne porte pas sur les inégalités dans la condition socio-économique des paysans, mais sur celles produites, à l'intérieur même des familles, par les mécanismes du système de reproduction sociale.

Le décès prématuré des parents, en perturbant le cycle d'accumulation et de redistribution des biens, constitue une première grande source de clivages et d'exclusion. Les difficultés économiques et l'émigration à l'extérieur du Saguenay entraînaient aussi un certain nombre d'abandons de l'agriculture. Mais, surtout, le fonctionnement régulier de ce système ouvert de transmission des biens accusait certaines inégalités: d'une part, entre les garçons et les filles et, d'autre part, entre les garçons selon les modalités respectives de l'établissement de chacun (donation du vieux bien, réallocation). Par ailleurs, le destin professionnel des exclus, qui s'avère généralement moins enviable, atteste selon l'auteur la validité de l'un des postulats de son enquête selon lequel le pluriétablissement est synonyme de réussite à la fois du point de vue du système de reproduction sociale et de la condition objective des établis.

À partir de ce critère de réussite (l'établissement dans l'agriculture), il devient certainement possible de déterminer un premier type de clivage entre les familles, entre celles qui produisent des exclus de l'agriculture (encore convient-il d'examiner la nature et les répercussions de cette exclusion) et celles qui réussissent une reproduction élargie dans l'agriculture. Mais comment, à partir de ce critère de l'établissement, évaluer le degré relatif de la réussite économique et sociale des uns par rapport aux autres? Que la majorité d'entre eux deviennent cultivateurs n'est pas vraiment surprenant à une époque où le destin de la majorité de la population demeurait lié à l'agriculture et à la société rurale. Mais, ce faisant, reproduisent-ils des statuts vraiment équivalents d'une génération à l'autre, d'une famille à l'autre? Les auteurs de plusieurs études de la paysannerie canadienne avant 1840 répondraient par la négative... en attendant des nouvelles du Saguenay, bien sûr.

Dans la seconde partie de l'ouvrage, G. Bouchard a présenté de manière très détaillée les objectifs et le fonctionnement du système de 
reproduction sociale saguenayen. Dans la troisième partie, la comparaison est à l'honneur. C'est ici que l'auteur fait une brève excursion en ville avant de partir à la recherche d'exemples extra-régionaux. Dans les deux cas, l'objectif est le même: vérifier si «sa» paysannerie pèche par originalité dans son mode de reproduction sociale. Dans le chapitre XIV, l'auteur jette un coup d'œil sur la reproduction sociale des familles ouvrières saguenayennes au $\mathrm{XX}^{\mathrm{e}}$ siècle. Cette étude s'appuie principalement sur un corpus d'entrevues portant sur 57 fratries suivies sur 2 et parfois 3 générations. Des informations qualitatives concernant 12 autres descendances s'ajoutent au dossier de cette enquête orale. Le fichier BALSAC, où sont disponibles des données sur le profil démographique, socio-économique et culturel des individus concernés, a aussi été mis à contribution.

L'analyse porte, on s'en doute, sur les modalités de la transmission des biens dans les familles initiales du corpus et sur le destin socioprofessionnel de leurs enfants. Les conditions de vie de ces familles étaient le plus souvent modestes. Leur fécondité relativement élevée, comparable à celle des autres familles ouvrières urbaines du Québec, entraînait une charge familiale plutôt lourde. De manière générale, ces familles perpétuaient, parfois même en les accentuant, les tendances égalitaires du mode de transmission de la paysannerie saguenayenne. C'était, à peu de choses près, la campagne en ville. Les ouvriers allaient jusqu'à reproduire le modèle paysan de corésidence et de service familial typique de la dynamique communautaire de la société rurale. C'est frappant, car les enfants des ouvriers ne pouvaient espérer, en retour de leur contribution «volontaire» envers la famille, une indemnisation comparable à celle des fils de paysans. Ne reposant pas sur des fondements strictement matériels, cette dynamique communautaire des familles ouvrières ne pouvait également s'appuyer sur une idéologie familiale aussi articulée que celle du monde paysan. L'auteur conclut cette première contre-épreuve comparative du système ouvert de reproduction en émettant l'hypothèse, fragile mais stimulante, d'une diffusion des tendances égalitaires du système de reproduction des campagnes saguenayennes, voire québécoises, vers les villes. Ce transfert aurait eu lieu au moment où les paysans délaissaient ces mêmes pratiques dans un contexte de saturation de l'espace agraire.

Dans le chapitre XV, la comparaison prend le large. G. Bouchard essaie d'étendre la portée du modèle saguenayen de reproduction, dit système ouvert, à l'ensemble des terroirs de peuplement du Québec et de l'Amérique du Nord, du moins aux régions rurales où a historiquement prévalu le modèle social de la petite paysannerie. Convenons-en, 
le pari est osé. Voyageant dans le temps comme dans l'espace, l'auteur puise dans la production scientifique relative à plusieurs sociétés rurales distinctes. Ces corpus historiographiques révèlent certaines préoccupations communes dans la définition des sociétés rurales précapitalistes, mais elles reflètent aussi en grande partie les enjeux idéologiques, variables d'historiographie en historiographie, du $\mathrm{XX}^{\mathrm{e}}$ siècle. Sont ainsi soumis à la visite virtuelle la vallée du Saint-Laurent avant 1800 et le Canada/Québec après cette date, auquel l'auteur associe la vallée du Haut-Saint-Jean (NB), le Canada anglais, le nord-est et le centre-ouest américain. Or, l'auteur trouve dans ces nombreux travaux, aux problématiques diverses et quelquefois contradictoires, une foule d'informations, souvent épurées de leur sens initial, pour construire des modèles de reproduction sociale comparables à celui du Saguenay. L'érudition de G. Bouchard est impressionnante et il démontre un talent indéniable de rassembleur de casse-tête. Mais l'exercice serait plus convaincant s'il se basait sur des études véritablement comparatives, auxquelles il ouvre d'ailleurs la porte.

En attendant ces travaux, l'image qui prend forme une fois ces pièces hétéroclites assemblées est celle d'une grande similitude entre les différentes sociétés en voie de colonisation, peu importe le lieu et le moment. Selon l'hypothèse de l'auteur, c'est seulement plus tard que les traits distinctifs de chacune deviendraient plus accusés. Évidemment, l'une sinon la principale des similitudes initiales est le modèle de reproduction familiale qui marque les débuts de toutes ces sociétés nord-américaines: le système dit ouvert de reproduction familiale, sujet du chapitre XVI.

À plusieurs reprises dans les chapitres précédents, G. Bouchard définit le modèle de reproduction saguenayen comme un système ouvert, typique des terroirs de peuplement récent de l'Amérique du Nord, en opposition avec les systèmes clos qui caractérisent les terroirs pleins de l'Europe. Dans ce chapitre, dernier de la troisième partie, il confronte plus directement cette opposition conceptuelle en essayant, à partir de travaux assez abondants et axés spécifiquement sur la reproduction des familles paysannes, de situer ses Saguenayens par rapport aux paysans français. Les conclusions de cet essai comparatif sont plus nuancées que celles du chapitre précédent. L'auteur insiste - peut-être un tantinet trop, car l'historiographie française, aux écoles de pensée bien définies, voit parfois des contrastes où il n'y en a pas - sur la complexité et la variété des modèles de reproduction sociale dans la France rurale. Le clivage terroir plein/terroir neuf n'est qu'un des axes assez nombreux de différenciation entre modes de reproduction. Plus révélateur encore, les terroirs de peuplement récent 
sont loin d'avoir l'apanage des tendances égalitaires et des solidarités familiales fortes.

Si ce chapitre tout en interrogations offre de quoi ébranler les amateurs de modèles trop schématiques, la dernière partie du livre risque de les déconcerter encore davantage. C'est ici que l'auteur s'interroge sur le pouvoir explicatif de la saturation des terroirs qui marque la fin du système ouvert. Le premier des trois chapitres de cette section, et dix-septième du livre, est pourtant assez rassurant. Il traite des répercussions du remplissage du territoire pour la reproduction sociale des paysans saguenayens. Dans une première section, l'auteur développe une méthode efficace pour déterminer, à partir de divers indicateurs démographiques et agraires, le seuil de saturation d'un territoire. Ensuite, il emploie cette méthode pour établir une cartographie très fine de la saturation de l'espace agraire pour l'ensemble des paroisses de la région de 1852 à 1971 et, plus loin dans le chapitre, pour l'ensemble de l'espace agraire québécois des $\mathrm{XIX}^{\mathrm{e}}$ et $\mathrm{XX}^{\mathrm{e}}$ siècles, à l'échelle plus générale des comtés. Grâce à ce lourd et ingrat travail de recherche, il peut vérifier les relations entre la dynamique de la saturation de l'espace et le passage du pluriétablissement à la reproduction «au singulier». Il peut également vérifier les impacts possibles de cette saturation sur la mobilité et la fécondité. Dans le premier cas, il y a des effets contradictoires; dans le second, la saturation ne semble pas expliquer grand-chose. Le doute est semé...

Après ce premier tour d'horizon, G. Bouchard s'attèle à l'analyse plus raffinée des rapports entre la saturation des terroirs et les autres changements affectant cette société rurale, avant d'esquisser un modèle plus général du changement social pour le Saguenay, voire pour l'ensemble du Québec. C'est le programme des deux derniers chapitres. Dans le chapitre XVIII, G. Bouchard examine donc le calendrier et les modalités de différents changements affectant la transmission des héritages et l'économie agricole. Il constate d'abord que la saturation agraire demeure un facteur important de changement social. En ce qui concerne les modalités de la donation, la terre devenue rare a favorisé un repli sur l'exploitation principale et sa transmission préférentielle à l'un des fils. Toutefois, les tendances égalitaristes sortent même renforcées au terme de ces modifications et le modèle paysan se rapproche alors de celui déjà décrit par l'auteur pour le milieu ouvrier urbain. L'un des enfants achète l'exploitation et les autres se partagent le produit. La saturation a par contre contribué à miner les fondements du service familial et à saper, ainsi, les bases matérielles et idéologiques de la dynamique communautaire. Mais, continue l'auteur, le rapport personnes/hectare n'agit pas seul, loin de là. Alors 
même que s'opérait au Saguenay cette saturation foncière, l'économie rurale se modifiait sous l'impact de la spécialisation grandissante de la production et des impératifs de la productivité. Ces changements compromettaient d'une autre manière le projet familial en s'attaquant aux fondements de l'ancienne économie «co-intégrée». Tout cela amène l'auteur «à nuancer substantiellement la thèse écologique et à lui accorder une place réduite dans l'explication des changements économiques et sociaux au sein de la paysannerie saguenayenne» (p. 431).

Et ce n'est que le début. Le dernier chapitre clôt sur une note encore plus surprenante cette enquête magistrale. À partir de l'analyse de vingt-cinq indicateurs démographiques, sociaux et culturels, G. Bouchard démontre l'impact relativement secondaire de la saturation des terroirs sur l'ensemble du processus de changement social. Les différents changements se produisent en ordre dispersé et selon un rythme variable qui, selon l'auteur, font douter de l'existence d'un moteur principal commandant le redéploiement des structures et des valeurs. Ce constat, contraire à l'un des principaux postulats initiaux de l'enquête, est révélateur de l'assurance et de l'humilité, au sens noble du terme, d'un grand chercheur conscient de l'ampleur et de la qualité scientifique du travail accompli, ainsi que de la richesse encore insoupçonnée que recèle le territoire de l'historien.

Voilà un aperçu du propos, ample à souhait, de Quelques arpents d'Amérique. Ce livre, nous l'avons dit, invite à voyager. Sur une gamme de sujets allant des migrations et de la transmission des héritages à la fécondité et à l'alphabétisation, il fournira des repères comparatifs pour l'histoire d'autres paysanneries, qu'elles soient proches ou moins proches du Saguenay dans le temps et dans l'espace. Souvent frustré devant les limites de sa démonstration, l'auteur appelle d'ailleurs de ses vœux ces comparaisons. Nous rejoignons l'intuition de départ du projet, à savoir que cette région peuplée sur le tard, offrant une bonne prise documentaire et même des centaines de récits de vie enregistrés - imaginez, dix-huitiémistes! - , pourrait servir de fenêtre sur des peuplements antérieurs et par là sur le (re)peuplement du continent. L'idée est séduisante et les comparaisons bien amorcées dans le livre. Il reste que l'auteur est de ces voyageurs qui se sentent chez eux partout; de la Nouvelle-France jusqu'au Minnesota, tout lui rappelle le Saguenay. Cette recherche de ressemblances, par ailleurs bien réelles, à la base même de la construction d'un modèle, est nécessaire. Mais n'est-ce pas à travers les différences que le modèle intègre l'histoire? Regardons comment ces Saguenayens habitent la durée. 
Revenons aux postulats de base. Au commencement, dit Gérard Bouchard, fut la famille, «acteur à partir duquel les données deviennent intelligibles» (p. 39). Il s'agit là d'une instance sociale que l'on pourrait qualifier de souveraine. Souveraine d'abord envers ses propres membres: rappelons-le, l'auteur va jusqu'à lui attribuer une individualité propre. Généralement prolifique, la famille se dote d'un projet, celui d'établir sur une terre agricole, directement ou par le biais du mariage, le plus grand nombre d'enfants possible. En échange, ceuxci se dépenseront volontiers. Ils contribueront de leur main-d'œuvre directement ou, s'ils travaillent pour autrui, en versant à la famille une bonne partie des gages, remboursables en entraide familiale lors de l'établissement.

Puissante vers l'intérieur, la famille ne l'est pas moins vers l'extérieur. Et fort heureusement, dirait-on, car elle doit faire face à un adversaire à sa hauteur: le capitalisme. C'est en effet un Capitalisme passablement envahissant que nous présente l'auteur, susceptible, si l'on le lui permet, de remodeler de fond en comble économie, société et culture. Or, au Saguenay, l'envahisseur est tenu en échec pendant près d'un siècle par personne d'autre que la famille, qui «[tient] tête pendant un temps aux sollicitations croissantes de l'économie et de la société capitaliste» (p. 423). Rusée, la famille fait entrer ce capitalisme dans une antichambre, lui prêtant ses fils pour le travail en forêt ou ailleurs ou en lui livrant un maigre surplus agricole sous forme de lait. Mais ce n'est que pour mieux l'enfermer. Au lieu d'embarquer pleinement dans la logique capitaliste, la famille lui préfère sa propre logique sociale (le pluriétablissement) qui exclut l'accumulation durable (L. Gérin ne disait-il pas déjà que «la race se décapite à chaque génération»?). Aussi, famille et capitalisme entretiennent-ils des rapports antagoniques mais fonctionnels, chacun profitant de l'autre, sans que soit compromis, et voilà l'essentiel, le projet familial. C'est cela, la co-intégration: la famille qui vit en ménage avec le capitalisme tout en gardant une invraisemblable virginité autarcique. Toute l'ambivalence de l'auteur envers son sujet se cristallise ici. Le comportement paradoxal de la famille est présenté à la fois comme une réussite et comme un signe d'une régression sociale et économique. «Ils y parviennent», souligne-t-il, trois fois plutôt qu'une (p. 137, 141, 142), au sujet du pluriétablissement, mais, ce faisant, Saguenayens semblent «[rater] le coche de la croissance capitaliste» (p. 128).

Toute bonne (?) chose a bien sûr une fin. Comme l'exige la cohérence, ce n'est pas en premier lieu le capitalisme qui aura raison de cette superbe familiale. Bien que l'auteur l'applique à des paysanneries européennes qui ne défrichent plus depuis longtemps, son modèle, 
un peu comme celui de Chayanov, ne s'épanouit pleinement qu'en contexte de peuplement. Finalement, c'est à la terre peu coûteuse (en argent, du moins) du front de colonisation, assise des stratégies de pluriétablissement, que la paysannerie saguenayenne doit son autonomie. Aussi le système est-il «[miné]... de l'intérieur» (p. 431) lorsque la terre vient à manquer: elle devient plus chère, encourageant par là l'entrée plus à fond dans l'économie de marché de ceux, même héritiers, qui doivent désormais l'acheter.

Cette famille, est-elle autre chose finalement qu'une magnifique machine à arrêter le temps? Tant que dure l'aubaine foncière, elle se contente de se reproduire, de se «cloner» à l'identique, comme l'ont fait avant elle beaucoup d'autres familles une fois lâchées dans la nature - pourtant déjà habitée - nord-américaine. L'auteur a beau exprimer des réserves tout à fait compréhensibles à l'endroit de la thèse étatsunienne de la «frontière», il en fait sien le schéma de base, ces cycles successifs mais interchangeables de peuplement. Il en épouse aussi la hantise de la fermeture, survenue au Saguenay à l'heure de la saturation définitive du territoire dans l'entre-deuxguerres. C'est alors que les Saguenayens paient le prix (ou récoltent les fruits?) de leur Wanderlust et de leur boulimie de terre boisée: «la fermeture des fronts pionniers... [les rend] vulnérables aux autres atteintes» (p. 432). C'est alors seulement, à partir des années 1940, qu'ils rejoignent l'histoire en marche. Ou, plutôt, c'est l'histoire qui les rejoint: dans un jeu complexe d'interférences étudié avec une candeur admirable dans les deux derniers chapitres, la région est enfin happée par les changements qui balaient la province. Avant, c'est le calme plat: la famille «retranchée» travaille à se reproduire socialement. Sommes-nous si loin que cela de la «folk society» ou d'autres «phases traditionnelles» chères aux sociologues?

Or, ces Saguenayens habitent peut-être leur front de colonisation, mais ils habitent aussi, dans un premier temps, le dix-neuvième siècle. L'analyse de l'auteur en fait très peu de cas. Le premier essor de l'industrie laitière? Elle aurait eu des effets négligeables, car elle ne correspond pas à la notion qu'a l'auteur, qui met la barre bien haute, du capitalisme conquérant. La hausse de la population non agricole, d'abord rurale (p. 180)? Elle ne change pas fondamentalement la donne reproductive, puisque ces non-établis, peu prisés d'ailleurs par ces agriculteurs bien agriculturistes qui ont l'oreille de l'auteur, ne représenteraient que les ratés du système. Et pourtant, tout cela signale l'intensification des échanges, la diversification des activités, l'accumulation (ou son contraire, tout dépendant des ressources de la famille). Ce sont là des signes que le capitalisme, à 'c' minuscule bien 
entendu, travaille lentement, de l'extérieur comme de l'intérieur, cette société. Il travaille ses familles, qui n'ont ici pas le luxe, là pas le désir de se retrancher, et pourquoi pas? ses individus, sûrement pas tous prêts à se sacrifier pour une «individualité» familiale que l'on devine paternelle. Les récits oraux (p. 303) attestent peut-être l'hégémonie de l'idéal du pluriétablissement, mais, dans les faits, les modèles ne sontils pas multiples, même en lisière de forêt?

Parions que ces Saguenayens en mouvement presque perpétuel ne se rapprochaient pas uniquement des limites de l'œkoumène, mais, petit à petit, du $\mathrm{XX}^{\mathrm{e}}$ siècle et de nous. Parmi les nombreux mérites de ce livre empreint d'ambivalence et de générosité, retenons celui de montrer les possibilités, mais aussi les limites de la thèse écologique.

\title{
QUELQUES ARPENTS D'AMÉRIQUE LES TENANTS ET ABOUTISSANTS D'UNE ENQUÊTE
}

\author{
GÉRARD BOUCHARD
}

Le long commentaire généreusement offert par les collègues Dessureault et Wien à propos de notre dernier ouvrage (Quelques arpents d'Amérique - à l'avenir: $Q A M$ ) invite à une double réflexion: en amont, sur ce que ce livre a voulu être et sur les voies d'analyse qu'il propose; en aval, sur les questions qu'il soulève et la recherche qu'elles appellent.

\section{A - Sur l'architecture du livre}

Rappelons d'abord brièvement les grands objectifs qui étaient poursuivis. Tout à fait dans l'esprit et même dans le prolongement du Village immobile (1972), nous visions à concrétiser les grands objectifs de l'histoire sociale, à savoir une démarche d'analyse qui a) tente de saisir l'ensemble des composantes du social, b) en mettant au jour leurs interactions c) dans une perspective de changement d) et en soumettant ses interprétations à l'épreuve de la comparaison. Pour y arriver, il fallait d'abord asseoir l'analyse sur un vecteur qui permette de couvrir tout le terrain, tout en respectant deux conditions: premièrement, éviter le procédé artificiel du «livre à tiroirs», qui juxtapose les composantes du social mais ne les articule pas vraiment les unes aux autres; puis, d'une façon ou d'une autre, effectuer le raccordement entre le microsocial (les individus, les familles, leurs stratégies...) et 
l'ordre sociétal ou macrosocial (ce que les sociologues appelaient naguère la société globale). Ce vecteur, nous avons cru le trouver parmi d'autres possibles - dans la reproduction familiale (à l'avenir: RF), assortie de tous ses paramètres géographiques, démographiques, économiques, sociaux et culturels (religion, alphabétisation, valeurs, etc.). Notons que ces paramètres ont été traités d'un double point de vue, à la fois comme variables indépendantes (ou comme facteurs déterminants de la $\mathrm{RF}$ ) et comme variables dépendantes, dans la mesure où elles étaient en retour infléchies ou modifiées par elle. C'est précisément ce double enchaînement qui assure le raccordement entre le micro et le macrosocial. À ces conditions, la dynamique complexe et englobante de la RF pouvait tenir sa promesse: constituer un fil conducteur, un axe d'où l'on puisse voyager ou «naviguer» dans le social. Mais on aurait tort de voir dans ce procédé analytique un parti pris quelconque octroyant à la famille une place «souveraine», comme le donnent un peu à entendre les auteurs de la Note critique.

D'où l'ordonnancement des chapitres et des parties du livre, qui met d'abord en place le territoire, la dynamique de la population, l'économie, les structures sociales (première partie), puis la reproduction familiale elle-même, dans toutes ses dimensions (deuxième partie), ensuite les comparaisons (troisième partie) et enfin l'analyse du changement social, dans une dernière partie qui confronte l'ensemble des variables étudiées et essaie de dégager un ordre, des convergences dans les diverses trames qu'elles dessinent. Les auteurs de la Note, à cause manifestement de l'espace restreint qui leur était imparti, ont dû faire des choix en attirant particulièrement l'attention sur une vingtaine de thèmes. Qu'on nous permette de mentionner ceux qui n'ont pu trouver place ou n'ont été qu'effleurés dans leur commentaire et qui contribuent néanmoins à soutenir l'architecture du livre: la problématique des collectivités ou populations neuves, la dynamique de l'enracinement des familles et de leurs descendants, le modèle (à quatre composantes) proposé pour l'analyse de l'économie agro-forestière, la critique de la proto-industrialisation et de la moral economy, la critique de Chayanov, de Gérin et de Miner, l'effort de conceptualisation et de modélisation en rapport avec la RF (aux fins de la comparaison, notamment: grille, typologie, etc.), le sort fait aux filles et aux femmes dans l'éducation, dans la transmission des biens et dans le mariage, la saturation de l'écoumène qui entraîne à la fois une différenciation collective et une accentuation des orientations égalitaires, l'éthique du service familial, les stéréotypes du paysan et de la société rurale canadienne-française et anglophone (et les fausses identités qui en résultent), le modèle élaboré pour expliquer la baisse de la fécondité, 
les problèmes théoriques inhérents à l'étude du changement. Cela dit, nous restreindrons nos remarques aux thèmes abordés par Dessureault et Wien et aux interrogations qu'ils soulèvent.

\section{$B$ - La reproduction familiale}

Après avoir rappelé avec beaucoup de soin et de clarté l'appareil méthodologique mis en place et les principaux résultats de nos analyses, les auteurs de la Note émettent des réserves sur les notions de réussite et d'échec de la RF. Nous avons conclu à une réussite lorsqu'un enfant était établi comme propriétaire-exploitant sur une terre neuve ou sur une terre déjà mise en valeur. Certes, les établissements effectués étaient inégaux et préparaient des destins variés, comme nous l'avons montré. Mais il nous a semblé que, dans un contexte de peuplement, l'accès (ou le non-accès) à la terre était un facteur déterminant. C'est ce qui poussait, par exemple, des avocats, médecins et notaires à exploiter une terre parallèlement à l'exercice de leur profession: le peuplement créait un contexte d'éloignement, de dénuement et d'insécurité matérielle contre lequel l'exploitation d'une terre même médiocre - offrait la meilleure des garanties (alimentation, chauffage, vêtement, habitat, productions pour le marché, etc.). Fautil rappeler que c'est précisément la recherche de terres qui a motivé la migration de toutes ces familles (parmi lesquelles, de nombreux journaliers et ouvriers) vers le Saguenay? Par ailleurs, les données orales redisent à l'envi l'importance que les parents attachaient à établir leurs enfants, d'une façon ou d'une autre, pour assurer leur sécurité, ce que confirment les statistiques tirées du fichier BALSAC. Même si un fils se retrouvait propriétaire d'une terre à peine défrichée, cette circonstance était provisoire et relevait d'une dynamique familière: le travail de la première génération garantirait l'aisance de la suivante et enclencherait un nouveau cycle de la $\mathrm{RF}$, par réallocation ou autrement ${ }^{1}$. Enfin, notre choix méthodologique ne suggère nullement que la qualité de l'établissement était un facteur non significatif dans l'analyse de la RF; mais dans ce contexte de peuplement (et donc d'éloignement), il était subordonné à un autre, plus déterminant dans l'immédiat, qui était le fait d'être établi ou non. C'est ce facteur que nous avons choisi de placer au cœur de notre étude (l'analyse des inégalités socio-économiques, de la mobilité et des rapports sociaux est en cours, dans le cadre d'un autre projet de recherche). Notre choix comportait un autre avantage: grâce au fichier BALSAC, il était pos-

1. Nous nous sommes expliqué sur ce point dans $Q A M$ (166-168). Le lecteur est également invité à se reporter au chapitre XIII où sont comparés les destins professionnels des enfants établis et non établis, afin de justifier la démarche adoptée. 
sible de suivre virtuellement l'ensemble d'une population (en l'occurrence, près de 10000 familles), plutôt qu'un échantillon.

En ce qui concerne l'analyse que nous avons faite d'un corpus de 306 donations, les auteurs de la Note nous reprochent d'avoir isolé cette pratique de transmission de toutes les autres, alors que, selon un des postulats du livre, les pratiques dévolutives doivent être étudiées dans leurs interrelations, en tant qu'elles forment un système complexe et dynamique. Nous comprenons peut-être mal l'objection. Le postulat n'interdit pas un aperçu particulier sur la donation, lequel nous paraît justifié à cause de a) la grande attention accordée à cette forme de transmission dans les travaux scientifiques, b) l'importance qu'elle prenait effectivement aux yeux des paysans, du fait qu'elle portait sur le «vieux bien», c'est-à-dire l'exploitation principale, c) certains malentendus dont elle est parfois l'objet; nous tenions précisément à faire ressortir, par exemple, que la donation ne représentait qu'un mode d'établissement parmi d'autres et qu'elle n'était pas synonyme d'inégalité. D'autre part, nos conclusions ne contiennent aucune inférence quant à la possibilité que la donation, telle que nous avons pu la reconstituer, soit typique ou non des contextes de peuplement ou de la région du Saguenay. Par contre, il nous semble que le cycle des avoirs fonciers que nous avons observé (formation de l'exploitation suivie d'une phase d'accumulation puis d'une phase de contraction ou de redistribution) avait plus de chance de se généraliser dans un contexte de peuplement où l'accès facile à la terre favorisait l'accumulation, tandis que les objectifs de la reproduction familiale y incitaient à la redistribution en faveur des nouveaux mariés.

Enfin, à propos de la diversité des modèles de RF, elle existait effectivement et nous pensons l'avoir mise en évidence dans les chapitres IX (par exemple: la répartition des familles selon la proportion de fils établis) et XIII (consacré à l'envers et aux revers de la RF). Cela dit, la réalité du pluriétablissement est massive, comme le démontrent bien les données statistiques du chapitre IX (aux premières décennies des fronts pionniers, plus du tiers des couples arrivaient à établir tous leurs fils; dans l'ensemble, les trois quarts des fils étaient établis, etc.).

\section{C - Les perspectives comparatives}

Pour mettre en perspective et mieux comprendre nos résultats sur la famille paysanne saguenayenne, cinq exercices comparatifs sont proposés, portant respectivement sur la famille urbaine au Saguenay, la paysannerie québécoise (hors Saguenay), le Canada anglais (hors 
Québec), quelques grandes régions des États-Unis et, enfin, la France. Plus d'une centaine de monographies sont ici mobilisées. En ce qui concerne en particulier la comparaison avec le Canada anglais et les États-Unis, l'enquête visait un objectif plus modeste (et plus prudent) que ne le laissent entendre les auteurs de la Note: il s'agissait en effet de nourrir la formulation d'une hypothèse, selon laquelle les contextes de peuplement se signaleraient par un certain nombre de traits collectifs communs. Les tendances égalitaires dans la transmission des avoirs font partie de ces traits communs, mais les ressemblances ne s'arrêtent pas là: la grille dont nous nous sommes servi pour mener la comparaison fait intervenir pas moins de 14 variables (tableau XV-1, $Q A M, 353)$. L'ampleur des similitudes relevées mérite réflexion, étant donné que, traditionnellement, les sciences sociales et historiques au Québec ont beaucoup insisté - en les prenant un peu pour acquises - sur les différences entre l'habitant canadien-français et ses voisins anglophones. Ce faisant, les chercheurs faisaient manifestement écho à une préoccupation des élites culturelles et politiques pour qui il était important de préserver la distinction, la spécificité (on dirait aujourd'hui: l'identité) canadienne-française. Nos analyses ont fait ressortir quelques-uns des stéréotypes et fausses représentations ayant résulté de ce paradigme. Dans ce contexte, la réflexion sur les ressemblances (indépendamment de toute volonté révisionniste ou «normalisante») s'annonce plutôt rafraîchissante comme exercice critique de vieilles représentations collectives et il ne nous presse guère, pour le moment, de retourner à la quête des différences.

Par ailleurs, notre hypothèse est formulée avec beaucoup de précaution dans le but d'éviter toute réduction déterministe qui inviterait à déduire de l'écologie du peuplement les traits des sociétés neuves. La comparaison avec les données françaises est éclairante à cet égard; on ne saurait dire, par exemple, si les tendances égalitaires saguenayennes et québécoises sont un héritage de coutumes françaises ou une caractéristique des terroirs neufs (un lien peut en cacher un autre...). Enfin, il est vrai que l'exercice comparatif avec les ÉtatsUnis serait plus convaincant s'il était lui-même appuyé sur un corpus d'études comparatives. Mais justement, un tel corpus n'existe pas; et pour cause.

\section{D - La co-intégration}

Ce modèle de la co-intégration constitue l'une des propositions principales du livre et il mérite qu'on s'y arrête parce qu'il est longuement commenté par Dessureault et Wien. De quoi s'agit-il? Il nous fallait rendre compte d'une réalité complexe, sinon paradoxale, dans 
la mesure où cette paysannerie saguenayenne a) entretenait des relations nombreuses et régulières avec le marché extra-régional mais sans vraiment se laisser convertir au capitalisme, b) manifestait simultanément des signes d'autonomie et de dépendance, c) faisait preuve de dynamisme et soutenait une vigoureuse croissance démographique et spatiale, mais s'inquiétait peu d'accroître sa productivité et de modifier en conséquence sa technologie et ses procédés, d) intégrait des changements tout en perpétuant d'importantes figures de permanence, e) vivait dans l'éloignement mais non forcément dans l'isolement, f) misait sur l'enracinement mais y parvenait au prix de la mobilité. Il nous a semblé dès le départ que l'arsenal des concepts et modèles disponibles en histoire économique et sociale ne permettait pas de rendre compte correctement de cette réalité. C'est pourquoi nous avons élaboré le modèle de la co-intégration. Essentiellement, il pose l'existence de deux systèmes ou deux dynamiques collectives, l'une locale (la société paysanne), l'autre extra-régionale (le capitalisme national, continental, international). Les deux systèmes entretiennent des rapports (souvent inégaux) de réciprocité en vertu desquels chacun assure son intégration et sa croissance en utilisant l'autre. Ainsi, l'économie capitaliste industrielle («moderne») tirait profit de la maind'œuvre peu coûteuse des campagnes pour faire tourner ses usines (à Québec, à Montréal ou en Nouvelle-Angleterre, par exemple), faire fonctionner ses chantiers forestiers, et le reste. En outre, le marché diffusait, à bas prix, les produits de la terre (lait, bétail, fourrages, bleuets, etc.). En retour, la famille paysanne tirait profit de ces maigres revenus pour restaurer ses équilibres précaires, perpétuer ses modèles de comportements (démographiques, par exemple), financer son expansion territoriale, etc. De ce côté, la co-intégration s'accompagnait d'importantes coordonnées économiques (la pluriactivité), sociales (le service familial) et culturelles (l'éthique de la solidarité) $)^{2}$.

À côté de la voie capitaliste et de la proto-industrialisation, la cointégration offre ainsi une autre voie pour penser le type de rapports que certaines collectivités paysannes ont entretenus avec le marché. Le modèle permet aussi de rendre compte des réalités complexes qui viennent d'être évoquées à propos du Saguenay - notamment le fait que pendant une cinquantaine d'années (et non pas un siècle, comme l'écrivent les auteurs de la Note), la famille paysanne a réussi à réa-

2. Précisons au passage: le service familial et la dynamique communautaire dont il relève ne sont pas synonymes d'harmonie ou d'absence de tensions et de conflits; ils expriment plutôt une situation objective d'interdépendance associée au contexte de peuplement et qui sert les intérêts de tout le monde. Dans certaines conditions exceptionnelles, ils peuvent s'en affranchir et, pour un temps, être portés surtout par la culture - comme il est arrivé au Saguenay après la saturation de l'écoumène et la fin du pluriétablissement. 
liser une forme d'autonomie (très relative, on le devine) au gré d'un fractionnement de ses dépendances (dans la pluriactivité). Dessureault et Wien s'en étonnent, parlent à ce propos d' «une invraisemblable virginité autarcique». Pourquoi «autarcique»? La co-intégration prend justement comme point de départ non pas une économie de subsistance mais un type de relations avec le marché. Pourquoi «virginité»? Nous sommes confrontés ici fondamentalement à une logique de réciprocité, d'ouverture, d'échanges, de mobilité, qui s'accompagne de divers changements dans la société, dans la culture et dans l'économie paysannes. Pourquoi «invraisemblable»? Nous donnons dans le livre de nombreux exemples de co-intégration dans diverses régions d'Amérique et d'Europe et l'analyse que nous en faisons pour le Saguenay est amplement nourrie de données empiriques. Il faudrait plutôt nous dire comment on pourrait expliquer autrement - et sans les nier - les importants indices de stagnation dans la productivité et la technologie agricoles, dans l'organisation et le fonctionnement des fromageries, sans oublier le maintien de la pluriactivité, du pluriétablissement, du service familial, de la fécondité naturelle? Dessureault et Wien se montrent particulièrement sceptiques sur ce point. Ce doute est légitime, certes. Mais il doit être apprécié en tenant un juste compte de l'armature théorique et du déploiement empirique qui soutiennent nos analyses.

$\mathrm{Au}$ sujet de la co-intégration toujours, Dessureault et Wien concluent: «Toute l'ambivalence de l'auteur envers son sujet se cristallise ici.» Cette ambivalence tiendrait dans le fait que l'analyse démontre à la fois la «réussite» de la co-intégration et la régression socio-économique qui l'accompagne. Or, c'est exactement ce que, en toute logique et très explicitement, nous avons voulu démontrer. La co-intégration suppose que les deux systèmes en interaction réalisent simultanément leur intégration et leur croissance mais selon des voies, des orientations, des modèles collectifs divergents. Dans le cas de la paysannerie saguenayenne, le coût à payer fut de s'installer dans une dynamique de reproduction qui divisait à chaque génération le capital et empêchait l'accumulation à long terme, qui perpétuait une civilisation matérielle et un genre de vie très rudimentaires, qui repoussait sans cesse le peuplement à la lisière de la forêt, sur des habitats fragiles et instables ${ }^{3}$. Si on étudie la RF de l'intérieur, dans ses ressorts et ses finalités déclarées, oui, on peut constater en toute objectivité

3. Nous avons parlé de régression pour caractériser cet aspect de la RF. Les auteurs de la Note n'aiment pas le concept, jugeant qu'il n'est pas neutre. Nous nous en sommes remis à la définition des dictionnaires. Régression: retour à un état antérieur. C'est exactement ce qui se produisait pour chaque nouvel établissement sur le front pionnier. 
qu'elle a «réussi». Si on l'évalue dans le prisme de la modernité capitaliste ou au regard du citadin d'aujourd'hui, on peut formuler une conclusion différente; mais elle ne relève pas de la même démarche (l'historien n'a pas à dire à ses acteurs qu'ils se sont trompés de société).

Trois autres critiques sont adressées au modèle et à l'utilisation que nous en faisons. D'abord, la définition que nous donnons du capitalisme, qui est jugée non conforme. C'est pourtant la plus classique que l'on puisse produire. Appuyée sur les références les plus autorisées, elle privilégie les traits suivants: commercialisation, profit et accumulation, spécialisation et productivitét. Or, la paysannerie cointégrée, c'est précisément le refus de l'accumulation continue, de la spécialisation et de la hausse soutenue de la productivité. En outre, selon les deux auteurs, notre analyse n'aurait pas fait justice à l'industrie laitière. Nous sommes ici au cœur de cette économie co-intégrée. Pour la très grande majorité des exploitants, le lait n'était qu'une source de revenu parmi plusieurs autres; ce revenu était géré dans le cadre et dans l'esprit de la pluriactivité et, avant 1935-1940, on n'a pas cherché à rationaliser cette production par des changements techniques, par une réforme de l'exploitation (voir, par exemple, la statistique des rendements laitiers, pratiquement stagnants entre le début du $\mathrm{XX}^{\mathrm{e}}$ siècle et 1940; sauf chez une minorité de producteurs, la saison de lactation n'a pas été étendue à l'hiver avant le milieu du siècle; on nourrissait les vaches avec de la paille durant la saison morte, etc.). C'est peut-être le fait principal, dont il faut rendre compte. En troisième lieu, selon Dessureault et Wien, notre analyse remettrait en selle la «folk society» et la société traditionnelle des sociologues. Disonsle franchement, nous sommes un peu gêné par une affirmation aussi lourde de conséquences qui survient au détour d'une phrase et laisse le lecteur sans explication. C'est faire peu de cas de toute la mise en place théorique et de toutes les précautions méthodologiques que nous avons prises afin de bien situer chaque point de notre démarche, afin de définir clairement chaque notion utilisée - précisément pour éviter ce genre d'anathème qui a fini par empoisonner il y a quelques décennies la réflexion sur la société paysanne québécoise et pour relancer la réflexion sur des bases plus rigoureuses. C'est faire fi, également, de l'effort de clarification théorique que nous avons fait en rappelant et en critiquant longuement les démarches de Gérin, de Miner, de la

4. Théoriquement, nous aurions pu aussi faire valoir le critère de la séparation de la propriété et du travail. Nous ne l'avons pas fait parce que, au Québec, même là où il s'est réalisé pleinement, le capitalisme agraire intégrait rarement ce trait. Il aurait donc été inapproprié de faire sur cette base le procès de l'économie paysanne saguenayenne. 
«moral economy», de Chayanov et autres. Faut-il rappeler que la «folk society» de Redfield est une représentation foncièrement évolutionniste, qui consacre un culturalisme primaire, qui pèche par anachronisme, qui véhicule des connotations tribales et en est venue à symboliser les pires stéréotypes de l'habitant canadien-français? Si les deux auteurs croient vraiment que nous avons donné là-dedans, ils doivent au lecteur une argumentation étoffée qui rende justice à la nôtre et qui donne vraiment prise à la discussion.

Deux autres remarques s'imposent à propos du modèle de la cointégration. Appliqué au Saguenay, il conduit à reconnaître un rôle important à la famille paysanne (ce que les deux auteurs traduisent à leur façon: «instance... souveraine», «au commencement était la famille»). Encore une fois, soulignons que le groupe familial est (dans ce type de collectivité) un lieu d'observation commode qui permet de parcourir une grande partie du terrain d'enquête. Il est en outre un agent important dans la structure sociale. C'est un statut que nous lui reconnaissons sans aucune hésitation: n'est-ce pas là l'un des grands enseignements de l'histoire sociale au cours des vingt-cinq dernières années ${ }^{5}$ ? Enfin, en ce qui concerne la dimension économique du modèle, il peut être utile de rappeler le vide qu'il veut combler. Dans la problématique de la transition des économies dites de subsistance vers le capitalisme, il veut rendre compte de tous ces itinéraires (ils sont très nombreux) qui ne suivent pas la ligne droite du type idéal capitaliste («classique») ou qui n'épousent pas la géométrie élégante et un peu capricieuse de la proto-industrialisation: toutes ces trajectoires qui font des nœuds ou changent de vitesse, qui prennent des formes hybrides, qui inventent des directions provisoires ou d'autres équilibres, et qu'on a vite fait de ranger parmi les marginalités, les anomalies, les résistances, les échecs. Ce sont en réalité des dynamiques collectives spécifiques dont les ressorts et le mouvement doivent être étudiés pour eux-mêmes, en évitant toute référence normative qui les disqualifie a priori. La co-intégration dessine l'une de ces configurations originales. Et c'est précisément le genre de service que la microhistoire sociale peut rendre aux sciences historiques: dans les interstices des grands modèles, mettre au jour d'autres modèles qui enrichissent la compréhension des premiers et qui attestent la diversité du changement.

5. T. K. Hareven le rappelait encore récemment devant la Social Science Association («What Difference Does It Make?» Social Science History, 20,2 (1996): 317-344). 


\section{E - Le changement social}

Allons vite car l'espace nous manque. Depuis vingt-cinq ans, de nombreuses études aux États-Unis et au Canada anglais ont montré que la saturation des espaces agraires entraînait un déclin de la fécondité et une redéfinition des comportements collectifs. Cette thèse néomalthusienne a pris une telle place qu'il nous a paru opportun de la vérifier au Saguenay, comme première hypothèse d'explication du changement - mais sans en faire un postulat de notre enquête. Finalement, il s'avère que la saturation a effectivement déstabilisé de l'intérieur la paysannerie saguenayenne et l'a rendue vulnérable à l'action de nombreux facteurs externes. Sous l'effet d'un enchaînement complexe de causes et suivant un calendrier à plusieurs vitesses, cette société a fini par basculer de la co-intégration à l'intégration, les années charnière coïncidant avec la Deuxième Guerre mondiale. Auparavant, plusieurs changements étaient survenus (certains très tôt, c'est même l'une des surprises de ce livre) mais la société les absorbait tant bien que mal - pensons à la hausse séculaire de l'alphabétisation, aux transformations dans les modes de choix conjugaux, à l'augmentation des déplacements grâce au chemin de fer, à l'accroissement de la taille des exploitations et du troupeau laitier, à l'augmentation du recours au crédit... On est assez loin du «calme plat» évoqué par Dessureault et Wien, ou de ce qu'ils appellent la «machine à arrêter le temps». Pour le reste, au Saguenay tout au moins, la diffusion de la contraception ne fut pas commandée de façon immédiate par la saturation. Il faudra voir ce qu'il en est dans d'autres régions du Québec, notamment dans les vieux habitats du corridor laurentien. Sur ce point, les auteurs de la Note simplifient un peu nos analyses, qui sont beaucoup plus prudentes et plus compliquées que ce qu'ils donnent à comprendre dans les derniers paragraphes de leur texte.

Les changements survenus entre 1920 et 1950 (par exemple, la baisse dans le recrutement des vocations religieuses dès après 1930) furent importants au point de fournir des éléments pour un nouvel éclairage sur la Révolution tranquille. Les transformations, qui sont aujourd'hui associées à cet événement et qu'on impute ordinairement à l'initiative d'élites éclairées, étaient en réalité souhaitées depuis longtemps par les classes moyennes et par les classes populaires rurales et urbaines. C'est une résistance au sein des élites qui les a retardées. La Révolution tranquille apparaît dès lors comme un rendezvous collectif où le peuple a peut-être précédé bon nombre de ses dirigeants... 
Terminons cette partie par deux remarques. Dans certains passages, Dessureault et Wien peuvent donner à penser que notre explication des permanences serait essentiellement de type culturel, et on reviendrait ainsi à la vieille thèse des mentalités réfractaires. Pour dissiper tout malentendu possible dans l'esprit du lecteur, il convient d'insister sur le caractère social - au sens le plus large — du modèle proposé. La culture y tient une place importante, bien sûr, mais comme une composante parmi d'autres, avec l'écologie, la démographie, l'économie, la structure sociale. C'est toute une dynamique collective qui est engagée dans la co-intégration. Du reste, c'est précisément sur ce terrain - du «culturalisme» - que nous critiquons la démarche de la moral economy. Dans d'autres passages, les auteurs de la Note suggèrent que les changements décisifs ont pu être le fait des enfants non établis et des citadins. C'est sans doute vrai de certains traits, par exemple les nouvelles habitudes de consommation. Du reste, notre analyse du changement accorde une place très importante aux facteurs dits externes. Mais au sein de ces facteurs, les influences extra-régionales l'emportent de loin sur l'action du segment non agricole dans la région. Il faut rappeler ici que les petites villes saguenayennes ont mis beaucoup de temps à se démarquer des campagnes (la différenciation ne survient vraiment qu'à partir de la décennie 1940) sous le rapport de l'alphabétisation, des pratiques religieuses, du service familial, des solidarités de parentèle, des modèles de nuptialité, de la fécondité, des conceptions prénuptiales. Quant à ceux et celles qui rompaient carrément avec la paysannerie et quittaient ses rangs pour une raison ou pour une autre (ils sont étudiés au chapitre XIII), il est difficile de dire dans quelle mesure ils ont pu altérer un système auquel ils n'appartenaient plus mais qu'ils continuaient à soutenir au moins en partie - nous avons pu le vérifier - notamment par l'entraide familiale. Enfin, au-delà du Saguenay et même du Québec, il y avait les fils et les filles qui voyageaient dans l'américanité; mais n'oublions pas que, pour la plupart, ces migrations de travail étaient commandées par la pluriactivité et assujetties à la règle du service familial: depuis l'Ontario et la Nouvelle-Angleterre, on veillait à retourner à la famille la plus grande partie des économies réalisées et ce, jusqu'au moment du mariage.

\section{$F$ - Petites mises au point}

Le texte de nos collègues Dessureault et Wien appelle d'autres précisions ou rectificatifs ad hoc. Pour la clarté du propos, nous en formulons quelques-uns, rapidement: 
- Sur les «plaisirs de la modélisation» et les emprunts à la sociologie «pour le meilleur ou pour le pire». Nous avons plutôt le sentiment d'avoir démoli pas mal de modèles sociologiques dans notre livre. Et d'une façon générale, nous nous sommes plutôt employé à introduire de la complexité et du non-prévisible dans les questions ou thèmes abordés (les dynamiques migratoires, l'économie agro-forestière, les processus de transmission, le rapport à la terre, le déclin de la fécondité, et le reste), comme le soulignent en d'autres passages les auteurs de la Note. Pour le reste, nous avons cherché à nous situer au cœur de l'histoire sociale, à la confluence de diverses disciplines, confronté à l'objet social dans toute son ampleur et sa diversité.

- Sur la thèse de la «frontière». Nous critiquons le modèle proposé par F. J. Turner mais demeurons attaché à la problématique des populations ou collectivités neuves. C'est un éclairage important qu'il nous paraît utile d'insérer d'une façon ou d'une autre dans l'étude des sociétés du Nouveau Monde. Si nous l'avons mal fait, nous sommes bien disposé à entendre une contre-argumentation. Mais une simple référence à la thèse de la frontière, de Turner (alors même que nous nous en distançons), ne suffit pas à disqualifier notre démarche. Pourquoi au juste ce rejet a priori d'une perspective qui paraît aller de soi?

- Sur le «rendez-vous différé». C'est l'analyse des données qualitatives (orales, principalement) qui est repoussée dans un prochain livre, et non pas l'étude socioculturelle. Celle-ci occupe déjà beaucoup de place dans le présent ouvrage.

- À propos de la comparaison avec la France. Les auteurs croient que la diversité des modes de RF y est exagérée. Il ne se trouvera pas beaucoup de spécialistes de l'Hexagone (français et autres) pour être d'accord avec eux. Par ailleurs, Dessureault et Wien insistent sur le fait que le clivage terroir plein/terroir neuf n'est qu'une clé parmi d'autres pour comprendre les différences entre le Québec et la France, et que les orientations égalitaires ne sont pas spécifiques au Nouveau Monde. Le lecteur aura peut-être l'impression que ce sont là des critiques qui nous sont adressées; ce sont en fait les principales conclusions de notre analyse comparative.

- Les grands changements dans l'économie paysanne. Ils sont survenus à partir de la décennie 1940 et non 1950.

- L'apport du travail forestier à l'essor d'une agriculture plus commerciale. Nous ne disons pas qu'il fut minime. Au contraire, le 
revenu forestier était investi dans l'exploitation et il a favorisé la conversion à l'industrie laitière. Mais cet apport fut minime dans l'essor du capitalisme agraire et dans le style de développement et d'accumulation qui lui est associé.

- La rareté croissante de la terre. Elle a favorisé une entrée plus à fond dans l'économie de marché, comme le soulignent les auteurs, mais, plus encore, le recours de plus en plus intensif à des pratiques économiques de type capitaliste (remembrement foncier, spécialisation, rationalisation...).

- Le degré d'autonomie atteint par la famille. Elle le devait pour beaucoup à la terre abondante, mais aussi à a) la pluriactivité, comme source de numéraire, b) la frugalité du paysan qui s'accommodait de gains relativement médiocres, durement acquis, c) la soumission des enfants à l'autorité parentale et à l'éthique familiale (prestations de travail sur la ferme, remise des gains réalisés hors ferme... $)^{6}$. On retrouve ici, encore une fois, la conjugaison de facteurs géographiques, économiques, sociaux, culturels.

- Les possibilités d'établissement sans le secours de la famille. Elles étaient restreintes. Un fils célibataire qui se soustrayait au service familial pour amasser un capital était plus ou moins rejeté par la famille. En outre, la mise en valeur des terres neuves, dans ses premières étapes, représentait un défi difficilement surmontable pour un individu isolé ou pour un jeune couple privé de l'assistance de la parentèle.

- Les tendances égalitaires de la RF en milieu paysan. Nous avons toujours assorti cet énoncé d'une importante réserve: le sort très incertain réservé aux filles.

- Sur les tendances égalitaires de la RF en milieu urbain. Nous nous sommes limité à poser la question de la diffusion du modèle paysan vers la ville. Nos résultats, non concluants sur ce point, ne nous ont pas permis d'en faire véritablement une hypothèse (QAM, 328-329).

\section{$G$ - Des voies, des problèmes de recherche}

Notre enquête saguenayenne soulève plusieurs questions à l'intention des historiens travaillant sur les autres régions du Québec rural, du $\mathrm{XVII}^{\mathrm{e}}$ au $\mathrm{XX}^{\mathrm{e}}$ siècle. Un peu paradoxalement, il est permis de

6. Sur ce point également, Dessureault et Wien expriment leur scepticisme. Il ne vient pas à propos: ce trait est l'un des mieux documentés de notre enquête. 
penser que les aperçus comparatifs présentés dans divers chapitres du livre contribuent à «désenclaver» cette paysannerie, en atténuant le caractère exceptionnel qu'on serait tenté de lui donner. Qu'il s'agisse de technologie et de procédés agraires, d'industrie laitière, de fécondité, de mode de reproduction familiale, de pluriactivité, de service familial ou de co-intégration, il y a gros à parier que les recherches comparatives à venir vont faire ressortir de nombreuses plages de similitudes avec le Saguenay, à côté, bien sûr, de contrastes importants. Il devrait en résulter une connaissance beaucoup plus nuancée de l'espace rural québécois ainsi que des processus et calendriers de diffusion du capitalisme. Faut-il appréhender ces résultats? Y a-t-il lieu de s'inquiéter d'une éventuelle prolifération du «syndrome» saguenayen dans diverses régions du Québec? L'enjeu est en réalité bien plus considérable. C'est au-delà du Québec qu'il faudra aussi porter la comparaison, pour y défaire le stéréotype idéal du paysan entrepreneur, «rationnel», capitaliste, bref, «normal»: en montrant que la co-intégration existait aussi dans des régions de l'Ontario, de la Nouvelle-Angleterre, du Midwest. Dans ces deux derniers cas, du reste, le travail est très avancé, grâce aux tenants de la family economy. Qu'on y regarde bien: notre idée invite à pratiquer une sorte de révisionnisme historique à rebours, sur le terrain de voisins qui ont souvent servi de références plus ou moins stéréotypées pour le Québec francophone, d'où il a résulté des réflexes de défense parfois mal inspirés.

C'est dire que, selon nous, l'aire d'application du modèle de la cointégration va bien au-delà du Saguenay et du Québec. Elle va bien au-delà aussi des paysanneries en contexte de peuplement; elle peut s'étendre à d'anciens habitats ruraux tout comme à des milieux urbains. Le modèle veut rendre compte d'un mode particulier d'intégration de certaines sous-populations (définies sur la base d'un clivage quelconque: spatial, économique, linguistique, religieux...) qu'on rejette ordinairement dans le fourre-tout des marginalités (d'une collectivité dite marginale, on sait ce qu'elle n'est pas mais non ce qu'elle est).

Notre livre invite aussi à une réflexion théorique et méthodologique sur l'analyse du changement social et les énormes difficultés auxquelles elle se heurte. Comment délimiter l'espace social à circonscrire tout en tenant compte de ses articulations externes? Lorsque les changements (économiques, démographiques, culturels...) ne s'alignent pas sur un même calendrier, comment en raccorder les trames? Et comment en démêler les causes? Sur l'histoire sociale elle-même, 
notre conclusion soulève des questions, émet des doutes et des inquiétudes aussi, tant ses objectifs sont exigeants et parfois très incertains. Tout cela est à mettre au compte sans doute de la relation entre l'historien et son objet: relation difficile, toujours à négocier, jamais assurée.

\section{H - Questions aux auteurs de la Note}

- Que devons-nous entendre exactement par l'expression: «ces nonétablis, peu prisés... par ces agriculteurs agriculturistes qui ont l'oreille de l'auteur»?

- La comparaison que nous avons faite avec les États-Unis a consisté à constater simplement l'attestation ou la non-attestation des 16 traits de la grille. L'exercice amenait parfois à mettre entre parenthèses la problématique et les objectifs spécifiques des travaux utilisés. Devons-nous comprendre que les résultats de la comparaison s'en trouveraient affaiblis, sinon compromis?

Nous terminons ces remarques en adressant des remerciements à nos collègues Dessureault et Wien, qui n'avaient pas une tâche facile. Ils devaient résumer, expliquer et commenter en quelques pages un gros livre qui propose un parcours parfois complexe et veut faire le tour de l'objet social. Ils s'en sont acquittés d'une manière très professionnelle en faisant une lecture fine de notre ouvrage, qui met en valeur le fond de l'enquête. Comme il est d'usage - sinon de règle - dans ce genre d'exercice proposé par la RHAF, nous nous sommes surtout employé à répondre aux objections, à défendre nos analyses, à en montrer les prémisses et les prolongements, à clarifier certains malentendus, à rectifier parfois ce qui nous a paru être des déformations. Mais il nous semble que l'exercice a produit ses fruits: les propositions principales de l'ouvrage sont bien en évidence, des questions sont soulevées, des critiques sont formulées, des avenues sont ouvertes. 


\section{RÉPLIQUE}

\section{CHRISTIAN DESSUREAULT THOMAS WIEN}

En plus de rappeler utilement les grands thèmes, l'architecture et un peu la genèse de son livre, Gérard Bouchard consacre une bonne partie de son commentaire à réagir à nos remarques. Nous l'en remercions. Mais cette réaction va dans un sens seulement: bien que généralement menée de façon «très professionnelle», notre critique n'en serait guère plus convaincante, du moins pour ce qui est des réserves formulées. Poliment mais fermement, Gérard Bouchard refuse de nous céder ne serait-ce qu'un seul de ses arpents d'Amérique... Sceptiques malgré tout devant certaines de ses analyses, nous saisissons l'occasion d'ajouter quelques brèves précisions.

1 - Vive le modèle, mais vive le modèle apprivoisé. En accordant à la reproduction familiale le statut enviable de «vecteur» de son analyse, G. Bouchard fait de ce phénomène social non seulement le premier critère du changement, mais aussi la finalité exclusive de ses Saguenayens. À notre avis, pourtant fondé sur l'étude de paysanneries de l'époque préindustrielle, c'est accorder trop d'importance et trop d'autonomie au social. Et surtout, c'est se montrer bien exigeant devant ce qui constitue un changement significatif. Sans vouloir nier l'importance des mutations du $\mathrm{XX}^{\mathrm{e}}$ siècle, au Saguenay comme ailleurs, nous plaidons pour un modèle moins binaire et moins schématique. Et lorsque nous évoquons la folk society, ce n'est bien sûr pas pour traiter l'auteur d'évolutionniste et encore moins d'historien paresseux. C'est pour souligner le lien de parenté entre son modèle et d'autres d'inspiration sociologique, friands de types idéaux et ayant de la difficulté à penser le changement autrement que comme une rupture brutale.

2 - Forte de ses réflexes paysans et pionniers, la famille saguenayenne décrite par G. Bouchard semble pouvoir s'isoler de son $\left(X^{2} X^{e}\right)$ siècle. Non pas qu'elle refuse le marché que lui propose le capitalisme, bien au contraire. Mais elle ne se laisse pas atteindre dans l'essentiel: elle conserve ses pratiques sociales témoignant, aux yeux de l'auteur, d'une rationalité précapitaliste. Chez Chayanov, c'était l'autarcie économique qui lui permettait de réaliser cet exploit; chez G. Bouchard, c'est la terre facilement accessible qui invite et oblige à 
la fois la famille à échapper au capitalisme envahissant. Ce manque de symétrie entre la participation réelle et croissante à divers marchés régionaux et suprarégionaux et les pratiques socioculturelles apparemment intouchées, nous avons voulu la saisir en parlant de «virginité» et de son contraire.

3 - L'auteur se réfère à plusieurs reprises aux témoignages oraux qui attesteraient la hantise d'indépendance et l'importance de l'éthique du service familial parmi les Saguenayens ruraux. Tout en lui enviant au plus haut point ces sources incomparables, nous nous posons quelques questions au sujet de ces témoignages «agriculturistes». Faudraitil s'attendre à une autre vision du passé de la part de ces informateurs survivants de la colonisation? N'est-ce pas justement au moment où elle apparaît menacée que l'indépendance familiale, au lieu d'aller de soi, prend les traits d'une idéologie? L'interrogatoire fut-il suffisamment critique pour filtrer les souvenirs teints de rose et pour débusquer les informateurs qui se montrent plus dévoués à la famille qu'ils ne l'étaient vraiment?

4 - Bien qu'étant au nombre des «citadins d'aujourd'hui» dont paraît se méfier l'auteur, nous partageons évidemment ses réticences devant des travaux fondés sur l'analyse condescendante de la marginalité. Il reste que les marges existent et que certains des indices de stagnation observés par l'auteur témoignent sans doute plus du manque d'enthousiasme du capitalisme, qui s'effrite quelque peu dans l'éloignement, que de celui des familles paysannes. En revanche, même dans l'éloignement, le capitalisme travaille sournoisement les sociétés régionales, selon des modalités qui varient dans l'espace et dans le temps. D'où nos réticences devant le vaste exercice comparatif de l'auteur. Ce modèle qui élit domicile en lisière de forêt marginalise son tour: il rélègue aux marges de son analyse les zones plus anciennes et plus développées de la région, avant de répéter l'exercice à l'échelle continentale. C'est une singulière colonisation à rebours. Pour nous, les pratiques pionnières célébrées par la thèse de la «frontière», même modifiée, nous enferment dans un exceptionnalisme stérile. D'ailleurs, si le pouvoir explicatif du paramètre de la «saturation» laisse à désirer, qu'en est-il de la «non-saturation» qui précède? Ce sont les pratiques paysannes, aux prises avec des changements à l'échelle mondiale ou du moins occidentale, qu'il faut situer dans leur vraie écologie.

Pour terminer, nous aimerions remercier Fernande Roy et son équipe, ainsi que notre indispensable adversaire, de nous avoir permis de participer à ce débat. Qu'il y en ait d'autres! 


\section{DERNIER MOT}

\section{GÉRARD BOUCHARD}

Étant donné les règles du débat, nous devrons nous en tenir à quelques brèves remarques. Nos deux interlocuteurs ainsi que les lecteurs voudront bien excuser le style télégraphique.

- Nous avons déjà dit dans quel esprit la reproduction familiale a été retenue pour être le vecteur de nos analyses. Mais, nulle part, nous n'en faisons «la finalité exclusive» de ces paysans. C'est toutefois une finalité de très grande importance.

- Nous aurions accordé trop d'autonomie au «social». Ce choix (qui ne devrait pas surprendre les praticiens de l'histoire sociale) s'accompagne partout dans notre ouvrage d'un souci de faire ressortir le rôle des autres variables, à la recherche soit d'interactions, soit de relations causales (par exemple: le chapitre XIX et l'analyse de régression).

- Nous aurions présenté le changement comme une rupture trop brutale. L'une des principales conclusions du chapitre XIX, c'est justement que le changement survient en vagues plutôt dispersées, suivant des calendriers déphasés. Cela dit, la série de nos indicateurs statistiques montre que les transformations les plus décisives en ce qui concerne le cœur de la co-intégration (institution de l'école obligatoire, fin de la colonisation, restructuration de l'économie agro-forestière et déclin de la pluriactivité, réforme agraire, diffusion de la contraception...) se concentrent effectivement en une vingtaine d'années, avec une décennie charnière, celle de la Deuxième Guerre. C'est ce qui fait basculer le vieux projet familial, dont les coordonnées socioculturelles, bien que déclinantes, survivront pendant un certain temps.

- À propos de la volonté parentale d'établir les enfants, nos sources orales refléteraient les «souvenirs teints de rose» de "survivants [d'une] colonisation... menacée». Voilà une audacieuse généralisation; la concordance qui se dégage de plus d'un millier d'entrevues (dont plusieurs très anciennes) serait à mettre au compte de la rationalisation. Une seule remarque à ce propos: les comportements de ces acteurs (voir nos mesures du pluriétablissement) s'avèrent en plein accord avec leurs paroles. 
— La famille paysanne saguenayenne s'isolerait de «son... siècle». Tous les contextes de peuplement entraînent un éloignement et une part d'isolement.

- Nous nous méfierions des «citadins d'aujourd'hui». L'énoncé est assez ambigu. Voyons-y une référence à la règle qui invite l'historien à se garder d'anachronisme en projetant indûment sur le passé ses jugements de valeurs.

- Le capitalisme s'effriterait dans cette région éloignée. Les perspectives et les occasions de profit qu'il y véhicule n'en sont pas moins réelles, comme nous l'avons montré (ex.: l'industrie laitière). Pourquoi n'en tire-t-on pas pleinement avantage plus tôt?

- La problématique des populations neuves enfermerait l'analyse «dans un exceptionnalisme stérile». Ainsi qu'il est arrivé dans toutes les collectivités des Nouveaux Mondes, le peuplement a été longtemps un horizon familier de la réalité et de l'imaginaire québécois. Comme partout ailleurs, la science historique doit en prendre acte. N'est-ce pas plutôt l'occultation de cette donnée qu'il faut craindre? Nous sommes peut-être ici au cœur du débat.

- Sur l'«exceptionnalisme» saguenayen: quelles sont les monographies locales ou régionales (du genre des Quelques arpents) qui fourniraient présentement les indispensables références comparatives à l'appui de cette thèse? Nous demeurons, quant à nous, ouvert à toutes les hypothèses; les travaux à venir trancheront. Mais on peut supposer qu'ils révéleront un espace rural assez hétérogène où le modèle «saguenayen» trouvera diverses résonances.

- Sur la «vraie écologie» des paysans saguenayens. C'était d'abord, nous semble-t-il, la terre à défricher, à proximité (ce pourquoi précisément ils avaient émigré). L'«échelle mondiale» venait après? 